\title{
Ecological surveillance of bat coronaviruses in Sarawak, Malaysian Borneo
}

\author{
Cheng-Siang $\operatorname{Tan}^{1 *} \mathbb{D}$, Vaenessa Noni ${ }^{1}$, Jaya Seelan Sathiya Seelan² ${ }^{2}$ Azroie Denel ${ }^{3}$ and Faisal Ali Anwarali Khan ${ }^{4}$
}

\begin{abstract}
Objective: Coronaviruses (CoVs) are natural commensals of bats. Two subgenera, namely Sarbecoviruses and Merbecoviruses have a high zoonotic potential and have been associated with three separate spillover events in the past 2 decades, making surveillance of bat-CoVs crucial for the prevention of the next epidemic. The study was aimed to elucidate the presence of coronavirus in fresh bat guano sampled from Wind Cave Nature Reserve (WCNR) in Sarawak, Malaysian Borneo. Samples collected were placed into viral transport medium, transported on ice within the collection day, and preserved at $-80^{\circ} \mathrm{C}$. Nucleic acid was extracted using the column method and screened using consensus PCR primers targeting the RNA-dependent RNA polymerase (RdRp) gene. Amplicons were sequenced bidirectionally using the Sanger method. Phylogenetic tree with maximum-likelihood bootstrap and Bayesian posterior probability were constructed.

Results: CoV-RNA was detected in ten specimens $(47.6 \%, n=21)$. Six alphacoronavirus and four betacoronaviruses were identified. The bat-CoVs can be phylogenetically grouped into four novel clades which are closely related to Decacovirus-1 and Decacovirus-2, Sarbecovirus, and an unclassified CoV. CoVs lineages unique to the Island of Borneo were discovered in Sarawak, Malaysia, with one of them closely related to Sarbecovirus. All of them are distant from currently known human coronaviruses.
\end{abstract}

Keywords: Bat, Cave, Coronavirus, Guano, Sarawak

\section{Introduction}

Coronaviridae consists of a large family of viruses that cause infections and diseases in a large range of vertebrates. Coronaviruses (CoVs) can be classified into four genera, Alphacoronavirus, Betacoronavirus, Gammacoronavirus, and Deltacoronavirus. Both Alphacoronavirus and Betacoronavirus houses CoVs of public health importance. Currently, there are seven known human CoV types, 2002/03 Severe Acute Respiratory Syndrome Coronavirus (SARS-CoV), 2012 Middle Eastern Respiratory Syndrome CoV (MERS-CoV), 2019 SARS-CoV-2, and four seasonal CoVs (HCoV-229E, HCoV-NL63,

\footnotetext{
*Correspondence: cstan@unimas.my

${ }^{1}$ Faculty of Medicine and Health Sciences, Universiti Malaysia Sarawak,

Kota Samarahan, Sarawak, Malaysia

Full list of author information is available at the end of the article
}

$\mathrm{HCoV}-\mathrm{OC} 43$ and $\mathrm{HCoV}-\mathrm{HKU} 1$ ) in which nearly all of them can be traced to their bat origin $[1,2]$. The discovery of two bats CoVs, KSA-287 from Taphozous perforatus and RaTG13 from Rhinolophus affinis with 100\% and 96\% sequence identity to MERS-CoV and SARS-CoV-2 respectively, sealed the hypothesis that these $\mathrm{CoVs}$ of Public Health Emergency of International Importance (PHEIC) indeed have originated from bats. However, the progenitor for SARS-CoV was never discovered.

The SARS-CoV epidemic started in Guangdong, China in 2002, rampaging through numerous countries for nine months, infecting 8096, claiming the lives of 774. A decade later, MERS-CoV emerged in the Kingdom of Saudi Arabia, but cases have mostly been reported around the Middle East, Africa and South Asia with occasional exported cases [3]. As of June 2021, there were 2564 confirmed cases of MERS, including 886 associated death, 
with the case-fatality-rate (CFR) of 34.4\%[4]. Later, a novel beta-coronavirus named SARS-CoV-2 was discovered in a cluster of patients with acute viral pneumonia of unknown origin in Wuhan, China. However, SARS-CoV-2 has a relatively low CFR of $2.1 \%$ compared to SARS-CoV and MERS-CoV but due to its high transmissibility, it has resulted in more than 208 million infections and 4.4 million deaths worldwide (as of 17th August 2021) [5].

Bats from the order Chiroptera, including microbat families Rhinolophidae, Hipposideridae, Emballonuridae, Miniopteridae, and Vespertilionidae are known to be the reservoir of bat CoVs in Asia [2]. These competent hosts can also be found in the touristic Wind Cave Nature Reserve (WCNR) in Bau District, Sarawak, Malaysian Borneo [6].

Other virological surveillance in Sarawak, Malaysia mainly revolves around viruses of public health importance, but the study on bat viruses in Gunung Mulu National Park, Sarawak ( $4^{\circ} 02^{\prime} 60.00^{\prime \prime}$ N $114^{\circ} 55^{\prime} 58.80^{\prime \prime}$ E), has led to the identification of Sarawak mobatvirus, a novel hantavirus within the genus mobatvirus [7]. Therefore, in the wake of the COVID-19 pandemic, it is timely that virological surveillance should be conducted to determine the presence and diversity of bat-CoVs in bat populations in Sarawak.

\section{Main text \\ Methods}

WCNR is located $1^{\circ} 24^{\prime} 55^{\prime \prime} \mathrm{N} 110^{\circ} 08^{\prime} 7^{\prime \prime} \mathrm{E}$ (Fig. 1). We have collected 21 pooled bat guano from WCNR on March 8th, 2021 and screened them for the presence of the partial RNA-dependent RNA polymerase gene of coronavirus. Five (5) pellets of fresh guano from insectivorous bats collected on a plastic tarp were pooled in $500 \mu \mathrm{L}$ of ice-cold viral transport medium (1st Base), vortex-mixed and centrifuged at $13,000 \times g$ at $4{ }^{\circ} \mathrm{C}$. Supernatant was used for nucleic acid extraction using High Pure Viral Nucleic Acid Extraction Kit (Roche). For phylogenetic analysis, the 440-bp gene of coronavirus was amplified using family-wide hemi-nested RT-PCR using the modified Watanabe primers: CoV-RVS3 5'-CCATCA TCASWYRAATCATCATA-3', CoV-FWD3 5'-GGT TGGGAYTAYCCHAARTGTGA- ${ }^{\prime}$ and CoV-FWD4/Bat $5^{\prime}$-GAYTAYCCHAARTGTGAYAGAGC- ${ }^{\prime}$. The complementary DNA synthesis was performed at $42{ }^{\circ} \mathrm{C}$ for 60 min using RevertAid Reverse Transcriptase (ThermoScientific) using CoV-RVS3. The conditions for the first round PCR were 2 min at $94{ }^{\circ} \mathrm{C} ; 40$ cycles for $20 \mathrm{~s}$ at $94{ }^{\circ} \mathrm{C}, 30 \mathrm{~s}$ at $50{ }^{\circ} \mathrm{C}, 30 \mathrm{~s}$ at $72{ }^{\circ} \mathrm{C} ; 1 \mathrm{~min}$. The conditions for the nested-PCR are identical except for the higher annealing temperature at $59{ }^{\circ} \mathrm{C}$. PCR amplicons were resolved in $2 \%$ agarose buffered in $1 \mathrm{X}$ TBE (1st Base)

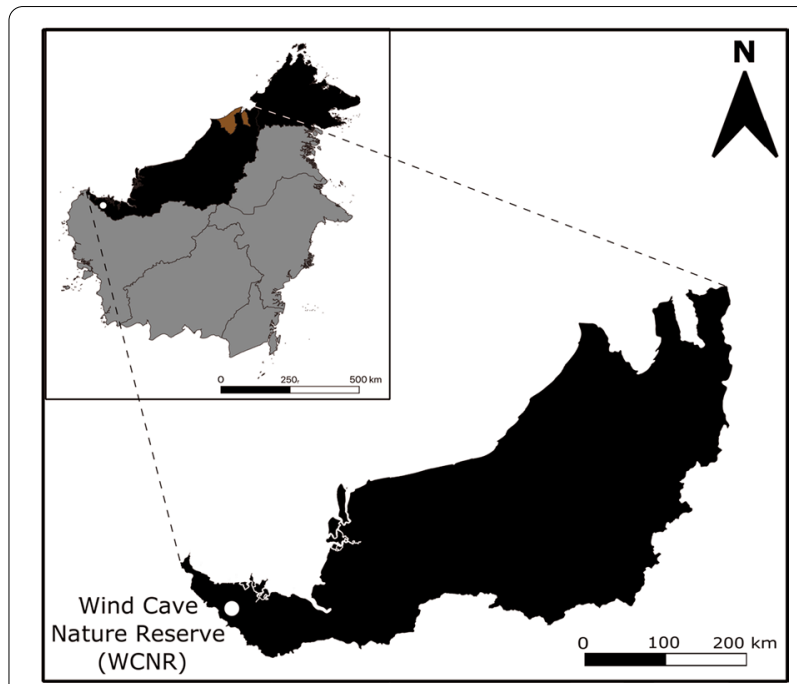

Fig. 1 Sampling location at Wind Cave Nature Reserve (WCNR), Sarawak, Malaysia Borneo. Three countries found on the Island of Borneo are differentiated with different colors: Black, East Malaysia; brown, Brunei; and grey, Kalimantan, Indonesia. The White dot represents the location of WCNR $\left(1^{\circ} 24^{\prime} 55^{\prime \prime} \mathrm{N} 110^{\circ} 08^{\prime} 7^{\prime \prime} \mathrm{E}\right)$ where guano specimens were sampled for virological surveillance. Map was created in QGIS Version 3.20.3 (http://qgis.org/en/site/)

supplemented with $10 \mu \mathrm{g} / \mathrm{mL}$ ethidium bromide (Promega), excised, and sequenced using the BigDye terminator v3.0. Amplicons were sequenced bidirectional using CoV-FWD3 and CoV-FWD4/Bat, amplicons with mixed or poor sequence read were ligated into pJet 2.1 vector and transformed into chemically competent Escherichia coli DH5 $\alpha$ (ThermoScientific) and sequenced using the vector primers (1st Base) [8]. Amplicon sequences were analysed with the Basic Local Alignment Search Tool software (BLAST) at the National Centre for Biotechnology Information (NCBI) to determine their corresponding species followed by nucleotide identity analysis using Sequence Identity and Similarity (SIAS) (http://imed. med.ucm.es/Tools/sias.html).

Sequence alignment of amplicon sequences and available CoV RdRp sequences from GenBank was carried out using MAFFT (https://www.ebi.ac.uk/Tools/msa/ mafft/). Phylogenetic tree was constructed based on 348 bp RdRp gene sequence with maximum-likelihood bootstrap/Bayesian posterior probability $\left(\mathrm{ML}_{\mathrm{b}} / \mathrm{PP}_{\mathrm{v}}\right)$ support using 1000 bootstraps and 1,00,00,000 $\mathrm{mcmc}$ generations respectively. The virus subgenus in the ML tree are categorized based on ICTV-approved genomes of all known Alpha (HKU10 isolate (MN312307), Alpha/R.fer/HuBCH/2013 (KJ473807), PEDV CV777 (AF353511), BtCoV/NL63-9b (KY073745), HCoVNL63 (NC0052645), HCoV-299E (NC002645), MkCoV/ WD1127 (HM245925), CCoV/Human/SwkMY/2018 
(MW591993), Alpha/Miniopterus/TH/B311-3/2008 (KJ020586) and BtCoV-HKU8/AFCF77(EU420139) and Beta-coronaviruses (Beta/Rousettus/CH/GCCDC1358/2014 (KU762338), MurineCoV-A59 (AY700211), HCoV-OC43 (AY391777), BetaCoV-HKU24-R05005I (KM349742), BtCoV-HKU5 (EF065509), HCoV-EMC (JX869059), BtCoV-HKU4 (EF065505), SARS-CoV-2 Wuhan-Hu-1 (MN908947), SARS-CoVr-2 RaTG13 (MN996532), SARS-CoV-1 Tor2 (AY274119), BtCoVSARSlike-CoV/SL-CoVXC45 (MG772933) and Beta/H. pra/ZhejiangCH/2013 (KF636752).

\section{Results}

The PCR positive rate was $47.6 \%(10 / 21)$ and with the distribution of $60 \%(n=6 / 10)$ and $40 \%$; $(n=4 / 10)$ alpha and beta-coronaviruses respectively. Bat CoVs identified in this study formed four novel monophyletic clades referred to as Borneo Beta-1 (GenBank: MZ574070, MZ574071), and Borneo Beta-2 (MZ574073, MZ574074), and Borneo Alpha-1 (MZ574065-MZ574067) and Borneo Alpha-2 (MZ574068, MZ574069, MZ573072) (Fig. 2).

Blast analysis revealed that Borneo Alpha-1 and Borneo Alpha-2 are most related to Alpha/Yunnan/ HIYN23/2020 (MZ081388) (81.96-83.76\% nt identity) and Alpha/H.dia/SbhMY/52/2013 (KX284940) (84.43-85.01\% nt identity) from China and Sabah (North Borneo) respectively. Whereas, Borneo Beta-1 and Borneo Beta-2 are most closely related to Beta/H.gal/ SbhMY/51/2012 (KX284939) (86.5-87.5\% nt identity) and Beta/H.pom/LAP/7521 (MN312609) (89.7-90.4\% nt identity) from Sabah state and and Laos respectively [2, 9]. Phylogenetic analysis revealed the close relationship of Borneo Alpha-1 and Borneo Alpha-2 with Decacovirus, specifically Decacovirus-1 and Decacovirus-2 respectively with strong $\mathrm{ML}_{\mathrm{b}} / \mathrm{PP}_{\mathrm{v}}$ support. On the other hand, Borneo Beta-1 is monophyly to Sarbecovirus, while Borneo Beta-2 is related to a currently uncategorised betacoronavirus.

\section{Discussion}

Despite the small sample size, the findings from this study confirm the presence of CoVs in nearly half of the bat guano sampled in WCNR, Sarawak, Malaysia. This supports the argument that bat guano is a good surrogate for Bat $\mathrm{CoV}$ surveillance without the need for life animal capture [10].

All of these CoVs were highly related to CoVs that were previously found in Hipposideros spp. suggesting that Hipposideros could potentially be their preferred natural host, but these viruses were also found in other bat species such as Rhinolophus, Miniopterus, Taphozous, Myotis, Scotophilus, and Cynopterus (fruit bats) [11-14]. However, SARS-CoV-2 related viruses were mainly identified in Rhinolophus bats such as $R$. affinis and $R$. malayanus in Yunnan, China, $R$. shameli in Northern Cambodia and R. acuminatus in Eastern Thailand [15].

Of the four novel clades, Borneo Beta-1 is probably of a greater concern as two members from its sister clade are responsible for two pandemics within the past two decades. Nonetheless, Borneo Beta-1 viruses are found to be distant to the SARS-CoVr-2 RaTG13 (MN996532) (69.1-69.7\% nt identity), the proposed progenitor of the pandemic SARS-CoV-2 identified in Rhinolophus affinis [16]. Further investigations to determine its receptor use should be carried out to assess the risk of spillover from bats to humans [17].

Caves are haven for ecotourism but may also serve as hotspots for bat $\mathrm{CoV}$ zoonosis since other sympatric wildlife is present and physical contact with viruscontaminated guano is nearly unavoidable. Although it is widely accepted that an intermediary host is almost always required for efficient spillover of Bat $\mathrm{CoV}$ to humans $[18,19]$, the discovery of CoV strain RaTG13 in Mojiang, Yunnan, China where fatal SARS-like epidemic in 2012 did not discount the possibility of direct spillover [20]. Following three CoVs zoonoses in the past 2 decades, ecotourism and research pertaining to caves and bats should be revisited.

There have been no records of bat $\mathrm{CoV}$ zoonoses in Sarawak. Nevertheless, it is important to note that clinical diagnostics of potential SARS-like/Influenza-like respiratory diseases in Sarawak had historically involved only bacterial diagnosis [21]. Virological surveillances were rarely carried out except through external research collaboration that would not impact patient care. Despite

\footnotetext{
(See figure on next page.)

Fig. 2 Phylogenetic tree of novel coronaviruses based on the nucleotide sequence of the partial RdRp gene. Maximum-likelihood tree of $348 \mathrm{bp}$ fragment of the RdRp gene from bat CoVs found in this study are colored according to their subgenera (Yellow = Borneo Beta-1 and Borneo Beta-2; Green = Borneo Alpha-1 and Borneo Alpha-2). Microhyla letovirus 1 (MLeV) (GVEC01031551) was used as the outgroup. Individual nodes are considered well supported when ML bootstrap values (BS) are at least $70 \%$ and Bayesian inference (PP) are at least $0.95 \%$ and tree with the highest log likelihood is shown (- 8794.58). Taxa are named according to the following pattern: Accension Number CoV Genus/Source or host/ Country/Lab code/Year. The following are the names in full of species, M.nip, Megaerops niphanae; C.sph, Cynopterus sphinx; C.bra, Cynopterus brachyotis; P.jag, Ptenochirus jagori; M.dau, Myotis daubentonii; P.cor. Pipistrellus coromandra; H.pom, Hipposideros pomona; H.gal, Hipposideros galeritus; H.pra, Hipposideros pratti; H.lar, Hipposideros larvatus; H.dia, Hipposideros diadema; H.lek, Hipposideros lekaguli; R.hip, Rhinolophus hipposideros; R.fer, Rhinolophus ferrumequinum; R.sha, Rhinolophus shameli; S.kuh, Scotophilus heathii and S.hea, Scotophilus heathii. Accension Numbers of positive bat guano samples are MZ574065-MZ574074
} 


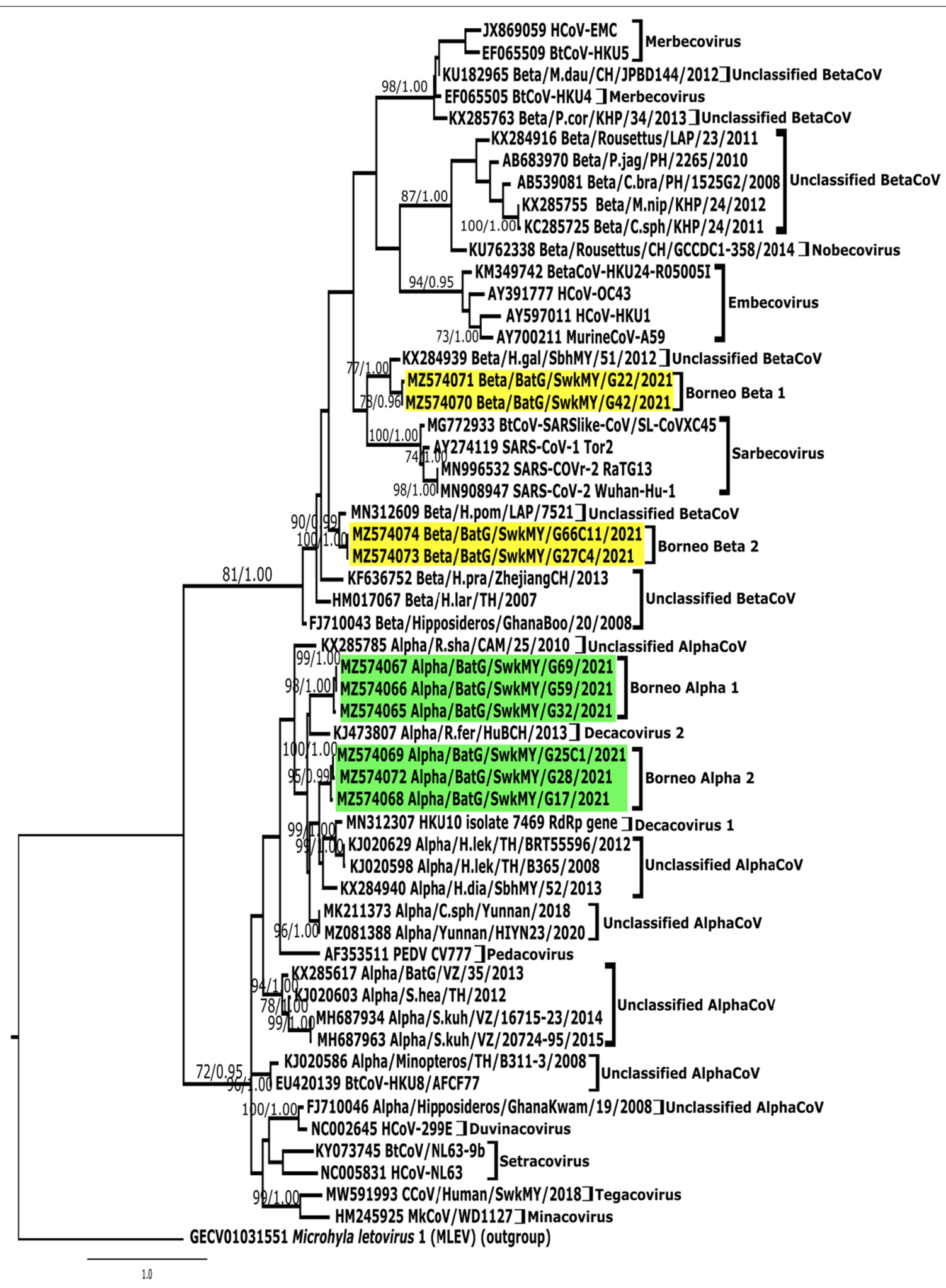

Fig. 2 (See legend on previous page.) 
the record adoption of real-time molecular assays in the healthcare settings during the SARS-CoV-2 pandemic, it was also shown that real-time assay was inferior to pan- $\mathrm{CoV}$ assay in the detection of seasonal $\mathrm{HCoV}$, missing out on many positives, including the novel canineCoVs in the human respiratory specimens [22, 23]. This is not surprising as real-time primers and probes tend to be more specific as compared to the degenerate primers used in most pan-CoV assays that increased the probability of detecting all circulating CoV genera. Do bear in mind that primer designs are largely based on the skewed representation of viral sequences obtained from other geographical locations, disregarding on the dispersal and vicariance phenomenon. To this, all SARS-like illnesses should be screened using the pan-CoV assay in hope of identifying the potential spillover rapidly and stop the epidemic at the source in a timely manner.

\section{Conclusions}

The bats in WCNR harbour rich diversity of both alphacoronaviruses and beta-coronaviruses that are unique to Borneo Island. Although these novel CoVs are not closely related to any seasonal, epidemic, and pandemic CoVs; visitors that are at risk of being in direct contact with virus contaminated guano. Basic biosafety precautions should be practised to minimize the possibility of spillover.

\section{Limitations}

The study was based on a small sample size obtained in a restrictive condition during the SARS-CoV-2 pandemic. Increasing the sample size may potentially lead to the discovery of more novel CoVs or quasispecies of the CoVs reported in this study. The partial RdRp sequence analysis may have suggested the novelty of the CoVs discovered in this study but longer sequence information on other genes and hopefully whole genomic sequences may enhance the current findings or suggest otherwise.

\footnotetext{
Abbreviations

CoV: Coronavirus; MERS: Middle eastern respiratory syndrome; nt: Nucleotide; PCR: Polymerase chain reaction; PHEIC: Public health emergency of international importance; RNA: Ribonucleic acid; SARS: Severe acute respiratory syndrome; WCNR: Wind cave nature reserve.
}

\section{Acknowledgements}

We would like to thank Sarawak Forestry Corporation for approving our sampling at WCNR (Ref: SFC.810-4/6/1-004 and Park Permit No. WL 33/2020). We also thank all the FAAK Lab members for their assistance in the field.

\section{Authors' contributions}

CST and FAAK participated in the design of the study and drafted the manuscript. VN and AD conducted the sampling, carried out the molecular genetic studies. CST, VN, FAAK and JS participated in the sequence alignment and phylogenetic analyses. CST, FAAK, JS and AD carried out revision of the manuscript. All authors read and approved the final manuscript.

\section{Funding}

Open Access funding provided by Universiti Malaysia Sarawak. This study was funded by the SEAOHUN Small Grant Program with the generous support of the American people through US Agency for International Development (USAID) One Health Workforce-Next Generation (OHW-NG) Award 7200AA19CA00018 and Malaysian Ministry of Higher Education, Fundamental Research Grant Scheme (FRGS/1/2019/WAB13/UNIMAS/03/2). Funders were not involved in the design of the study and collection, analysis, interpretation of data and writing the manuscript. The contents and associated materials are the responsibility of the authors and do not necessarily reflect the views of USAID or the US Government.

\section{Availability of data and materials}

All bat CoV nucleotide sequences obtained in this study can be accessed in GenBank (https://www.ncbi.nlm.nih.gov/) under the Accession Number MZ574065-MZ574074.

\section{Declarations}

Ethics approval and consent to participate

Not applicable.

Consent for publication

Not applicable.

\section{Competing interests}

The authors declare that they have no competing interests.

\section{Author details}

${ }^{1}$ Faculty of Medicine and Health Sciences, Universiti Malaysia Sarawak, Kota Samarahan, Sarawak, Malaysia. ${ }^{2}$ Institute for Tropical Biology and Conservation, Universiti Malaysia Sabah, Kota Kinabalu, Sabah, Malaysia. ${ }^{3}$ Sarawak Forestry Corporation, Kota Sentosa, Sarawak, Malaysia. ${ }^{4}$ Faculty of Resource Science and Technology, Universiti Malaysia Sarawak, Kota Samarahan, Sarawak, Malaysia.

Received: 24 September 2021 Accepted: 6 December 2021

Published online: 20 December 2021

\section{References}

1. Memish ZA, et al. Middle east respiratory syndrome coronavirus in bats, Saudi Arabia. Emerg Infect Dis. 2013;19(11):1819.

2. Anthony SJ, et al. Global patterns in coronavirus diversity. Virus Evol. 2017. https://doi.org/10.1093/ve/vex012.

3. Zaki AM, van Boheemen S, Bestebroer TM, Osterhaus ADME, Fouchier RAM. Isolation of a novel coronavirus from a man with pneumonia in Saudi Arabia. N Engl J Med. 2012;367(19):1814-20. https://doi.org/10. 1056/NEJMoa1211721.

4. WHO EMRO|MERS outbreaks|MERS-CoV|Health topics. WHO. 2020. http://www.emro.who.int/health-topics/mers-cov/mers-outbreaks.html. Accessed 19 Aug 2021.

5. COVID Live Update: $208,720,496$ cases and 4,383,997 deaths from the coronavirus - worldometer. 2021. https://www.worldometers.info/coron avirus/. Accessed 17 Aug 2021.

6. Ridwan M, et al. Bats of the Wind Cave Nature Reserve, Sarawak, Malaysian Borneo. Sarawak: WCNR; 2011.

7. Zana B, et al. "Molecular identification of a novel hantavirus in Malaysian bronze tube-nosed bats (Murina aenea). Viruses. 2019. https://doi.org/10. 3390/v11100887.

8. Latinne A, et al. Origin and cross-species transmission of bat coronaviruses in China. Nat Commun. 2020. https://doi.org/10.1038/ s41467-020-17687-3.

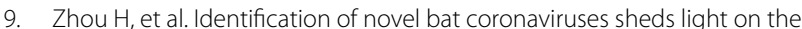
evolutionary origins of SARS-CoV-2 and related viruses. Cell. 2021. https:// doi.org/10.1016/j.cell.2021.06.008. 
10. Watanabe $\mathrm{S}$, et al. Bat coronaviruses and experimental infection of bats, the Philippines. Emerg Infect Dis. 2010;16(8):1217-23.

11. Bourgarel M, et al. Circulation of Alphacoronavirus, Betacoronavirus and Paramyxovirus in Hipposideros bat species in Zimbabwe. Infect Genet Evol. 2018;58:253-7.

12. Wacharapluesadee $S$, et al. Diversity of coronavirus in bats from Eastern Thailand emerging viruses. Virol J. 2015;12(1):1-7.

13. Wacharapluesadee $\mathrm{S}$, et al. Evidence for SARS-CoV-2 related coronaviruses circulating in bats and pangolins in Southeast Asia. Nat Commun. 2021;12(1):1-9.

14. Han Y, et al. Identification of diverse bat Alphacoronaviruses and Betacoronaviruses in China provides new insights into the evolution and origin of coronavirus-related diseases. Front Microbiol. 2019. https://doi.org/10. 3389/fmicb.2019.01900.

15. Hassanin A, Tu VT, Curaudeau M, Csorba G. Inferring the ecological niche of bat viruses closely related to SARS-CoV-2 using phylogeographic analyses of Rhinolophus species. Sci Rep. 2021;11:1-11.

16. Zhang $\mathrm{YZ}$, Holmes EC. A genomic perspective on the origin and emergence of SARS-CoV-2. Cell. 2020;181(2):223-7.

17. Wang LF, Anderson DE. Viruses in bats and potential spillover to animals and humans. Curr Opin Virol. 2019;34:79-89.

18. Wang $M$, et al. SARS-CoV infection in a restaurant from palm civet. Emerg Infect Dis. 2005;11(12):1860-5.

19. Zhang $\mathrm{S}$, et al. Bat and pangolin coronavirus spike glycoprotein structures provide insights into SARS-CoV-2 evolution. Nat Commun. 2021;12(1):1-12.

20. Zhou P, et al. A pneumonia outbreak associated with a new coronavirus of probable bat origin. Nature. 2020;579(7798):270-3.

21. Toh T-H, et al. High prevalence of viral infections among hospitalized pneumonia patients in equatorial Sarawak, Malaysia. Open Forum Infect Dis. 2019. https://doi.org/10.1093/ofid/ofz074.

22. Vlasova AN, et al. Novel Canine coronavirus isolated from a hospitalized pneumonia patient, east Malaysia. Clin Infect Dis. 2021. https://doi.org/10. 1093/cid/ciab456.

23. Fieldhouse JK, et al. Panspecies molecular assays detect viral pathogens missed by real-time PCR/reverse-transcriptase PCR among pneumonia patients, Sarawak, Malaysia. Trop Dis Travel Med Vaccines. 2020;6(1):1-8.

\section{Publisher's Note}

Springer Nature remains neutral with regard to jurisdictional claims in published maps and institutional affiliations.

Ready to submit your research? Choose BMC and benefit from:

- fast, convenient online submission

- thorough peer review by experienced researchers in your field

- rapid publication on acceptance

- support for research data, including large and complex data types

- gold Open Access which fosters wider collaboration and increased citations

- maximum visibility for your research: over $100 \mathrm{M}$ website views per year

At $\mathrm{BMC}$, research is always in progress.

Learn more biomedcentral.com/submissions 